\title{
LINEAR VIBRATION ANALYSIS OF FUNCTIONALLY GRADED THICK BEAMS CARRYING POROSITIES UNDER THERMAL LOADS
}

\section{IBNORACHID ZAKARIA, BOUTAHAR LHOUCINE \& EI BIKRI KHALID}

Research Scholar, Mohammed V University, Rabat-ENSET, M2SM, Morocco

\begin{abstract}
In the current research, the vibrational behavior in bending of FG beams carrying porosities (PFGBs), exposed to different thermal loads is studied according to an efficient beam theory; the displacement field is determined by a hyperbolic variation of transverse andin-plane displacements through the thickness. The number of unknowns and, consequently the number of governing equations is reduced by decomposing the transverse displacement into shearing and bending components.On PFGB lower and uper faces, the boundary conditions are respected without shear correction. Material mechanical and thermal characteristics depend on the temperature, and change with beam thickness according to a modified mixing law. Even and uneven porosities-distributions through the beam cross-section are used. Hamilton's principle is applied to obtain the motion equations. Boundary conditions Hard and Simply Supported (HSS) are considered to allow beam-buckling. The differential equations system is solved by using the Navier-solution. The temperature and porosity effects on the frequency responses and buckling of HSS-PFGB are shown graphically and numerically, and then discussed in detail.

KEYWORDS: Functionally Graded Beam, Porosity, Thermal environment \& Critical buckling temperature
\end{abstract}

Received: Apr 23, 2020; Accepted: May 14, 2020; Published: Jun 06, 2020; Paper Id.: IJMPERDJUN202086

\section{INTRODUCTION}

In laminate structures, the lamellae are assembled to improve their thermal and mechanical characteristics. But, the abrupt change in materials characteristics at the interface between both layers inducing important inter-laminar stresses which can lead to delamination and cracking until damaged of structure. These defects are corrected by the new composite materials with functional gradation (FGMs) which have characteristics varying across a desired direction, according to the mixing ratio of their constituents.For this type of materials, there is no concentration of stresses observed in the laminated structures. FGMs are generally composed of a mixture of ceramic and metal. Ceramic has a lowthermal-conductivity, which allows it to resist at high temperatures and to prevent metaloxidation. Metal with its ductile, avoids ruptures caused by thermal constraints.In addition, ceramic and metal are easy to mix with a constantly changing proportion [1].

Evolution of FGMs has solicited the attention of researchers and engineers, due to their great resistance to very hot environments. This explains their increasing use in aeronautical and aerospace engineering (reactors nuclear, rocket nozzles and engine components).Thus, it is necessary to perform precise analyses on the behavior of FGM structures working in the environments with high temperatures.Many theories are therefore developed to study and analyze the different structures. References [from 2 to 18] present research carried out in last years on different FGM structures to analyse their dynamic behavior in vibration.Several studies on thermal behavior of FG beams are available in the literature.State space analytical model was presented by Trinh et al [19] to analyse dynamic behavior of FG beams exposed to mechanical loads in hot environment, with different boundary 
conditions. A mathematical analysis was introduced by El-Megharbel [20] to investigated bending vibration of FG beam heated, considering two heat distributions along the structure. Buckling in thickness and length directions of a FG beam was investigated by Şimşek [21] using Timoshenko Beam Theory and Ritz method.

A thermal behavior analysis of functionally graded beams has been carried out by Davoodinik et al [22].Ma and Lee [23] deduced the equations of motion for both static dynamic behaviors of FGBs under a linear temperature field. As for Gaetano Giunta et al [24], they investigated mechanical behaviour of 3D-beams exposed to a nonlinear temperature field.

Sintering is to consider one of the most practical methods of manufacturing FG materials. The material thermal characteristics of the components are not the same, therefore their solidification during the sintering process is also different, and thus porosities can occur through the material [25]. This is why it's necessary to take into consideration the impact of porosity during the analysis and design of structures in FG materials.Moreover, pores produced in the FG structures make them light and increase their rigidity [26].

Studies concerning vibrational responses of FGM structures carrying porosities are not many.Nuttawit Wattanasakulpong and Variddhi Ungbhakorn [27] studied FG beam behavior in bending free vibration by assuming arbitrary boundary conditions, introducing elastic ends.DTM and Timoshenko's model were used by Ebrahimi and Mokhtari [28] to analyze flexural vibrational responses of FG beam carrying porosities.

Chebyshev-collocation method is used by Nuttawit Wattanasakulpong and Arisara Chaikittiratana [29] to analyse dynamic behavior of FG Timoshenko beam containing porosities.By applying the refined theory, Hassen Ait Atmane et al [30] studied the impacts of normal deformation and porosity on vibrational behavior of functionally graded beams on various elastic bases. The first study on FG beams carrying porosities and working in a hot environment was carried out by Ebrahimi and Salari. [31] to show porosity distribution-effects on beam-vibrational behavior.Farzad Ebrahimi and Ali Jafari [32] studied the facts of temperature on the flexural vibration of the Timoshenko and Reddy FG beams with porosities.They adopted the Navier solution and the Reddy beam model in a second work [33], to study the dynamic behavior in bending of FG beams containing porosities. A mechanical loading in a high temperature environment is assumed.

The aim of this work is to analyze vibrational-responses of a thick beams carrying porosities and considered in different hot environments. Both porosity-distributions are proposed. Refined higher order beamtheory has been used.The mechanical and thermal characteristics vary across the thickness direction of the beamversus temperature according to a modified mixing law. Three fields of temperature, uniform, linear and nonlinear-sinusoidal are proposed, and the both porosity distributions in the same direction, even and uneven are added. In this theory, axial displacement varies in the thickness direction of the PFG beam according to a higher order function, without any shear correction. Hamilton's principle is applied to obtain motion-equation, and Navier's solution is adopted to solve obtained equations system. Numerically and graphically results are presented to highlighting the influence of temperature, porosity and their variations across the beam-thickness on thermal buckling behavior and frequency responses of HSS-PFGB.

\section{BASIC THEORY AND FORMULATION}

\subsection{Study Structure Definition}

The Porous FG beam investigated here has length $L$ and rectangular cross-section $b \times h$, with $h$ being the height and 
$b$ being the width as shown in figure 1.Beam upper face is entirely ceramic, while the lowerface is completely metallic. Composition of both constituents varies continuously between the two faces, depending on the volume portion.

Variation of the beam material characteristics $(C=\rho, E, v, \alpha)$ through its thickness depends on the material parameter $(p)$ which characterises the volume portion of each beam material constituent, the distribution-type of porosity and the index of porosity $\zeta \ll 1$.Beam is considered perfect if $\zeta=0$. Even and uneven porosity-distributions are proposed here, and presented in the same figure 1.

$\rho$ : Mass-density. E: Young's modulus. $v$ : Poisson's ratio. $\alpha$ : Thermal-Coefficient.

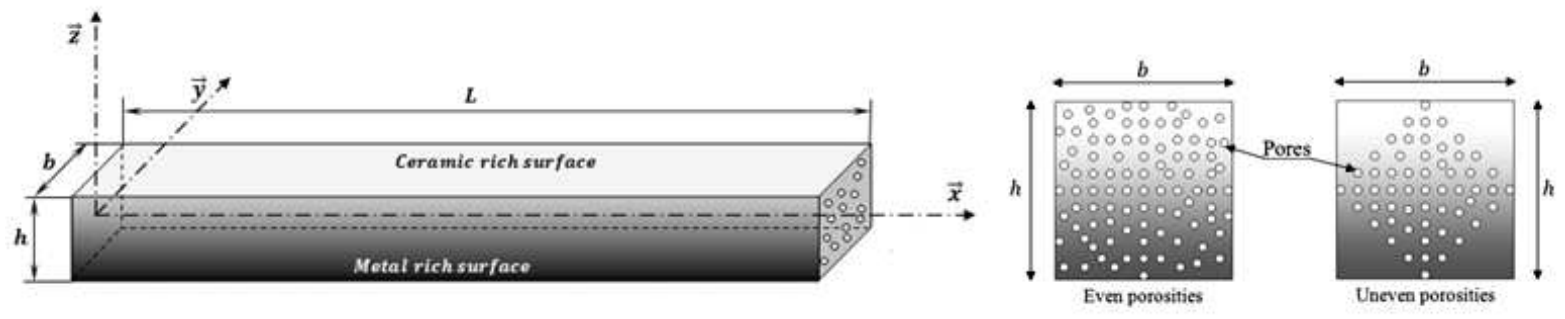

Figure 1: PGFBeam - Geometry, coordinates and both Porosity Distributions.

Figures 2 (a) and (b) show the Young's modulus distributions through PFG beam-thickness for certain values of $p$ and in both distribution of porosities cases. A constant decrease in this module is shown in Figure 2 (a) for the porosity even-distribution case. While, in uneven-distribution of porosities case, a concentration of porosities in beam-cross section middle-area is located, as shown in Figure 2 (b). This leads to a strong reduction in Young's module.
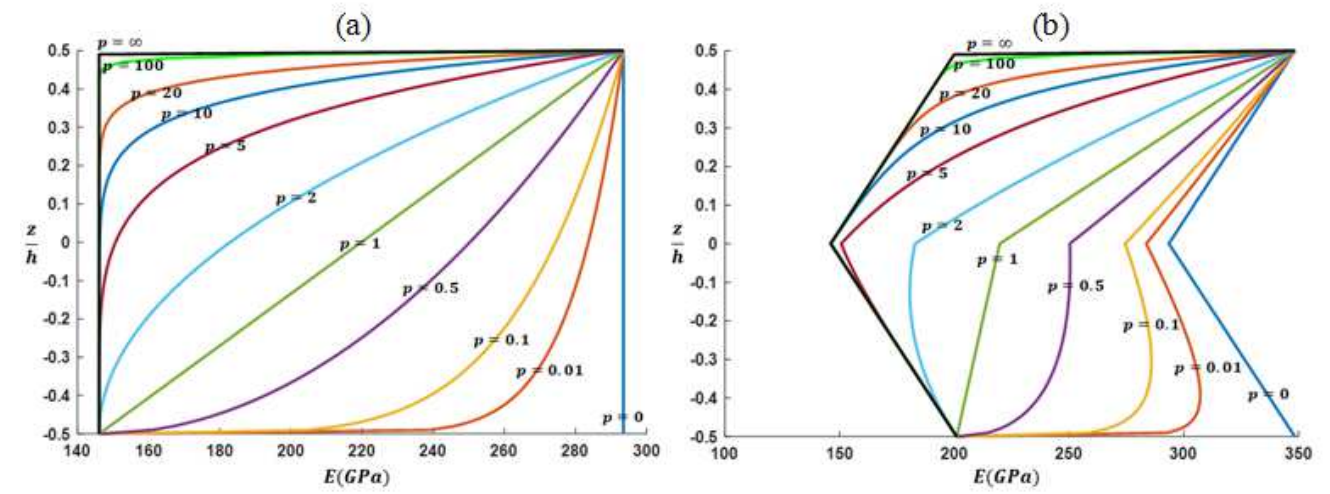

Figure 2: Effect of the Porosity-Distribution type, (a): Even, (b): Uneven, on the Young's Modulus, for some values of $(p)$. $(\zeta=0.2)$

\subsection{Proposed Thermal Environment}

In very hot environments, strong changes in the mechanical and thermal characteristics of FGMs can be anticipated [34]. These characteristics $(C=\rho, E, v, \alpha)$ are supposed to be related to temperature by the following relation [35]:

$$
C(T)=C_{0}\left(C_{-1} T^{-1}+1+C_{1} T+C_{2} T^{2}+C_{3} T^{3}\right)
$$

where $C_{0}, C_{-1}, C_{1}, C_{2}$ and $C_{3}$ are the thermal coefficients given in Table1 for ceramic $\mathrm{Si}_{3} \mathrm{~N}_{4}$ and metalSUS304 [36]. PFG beam-lower face $(z=-h / 2)$ is completely metallic, and uper face $(z=+h / 2)$ is purely ceramic.

Effective characteristic of the material $C$ depends, both on the characteristics of each constituent $C_{m, c}$, on the 
temperature $T$ and on the position $z$. In the even porosity-distribution case, this characteristic is defined as:

$$
C_{\text {even }}=\left(C_{c}-C_{m}\right)\left(0.5+\frac{z}{h}\right)^{p}+C_{m}-\frac{\zeta}{2}\left(C_{c}+C_{m}\right) ; \quad C=\rho, E, v, \alpha
$$

and in uneven porosity-distribution case as:

$$
C_{\text {uneven }}=\left(C_{c}-C_{m}\right)\left(0.5+\frac{z}{h}\right)^{p}+C_{m}-2 \zeta\left(2-\frac{|z|}{h}\right)\left(C_{c}+C_{m}\right) ; \quad C=\rho, E, v, \alpha
$$

Table1: Thermal Coefficients of the Mechanical and Thermal Characteristics of both PFG Beam MaterialConstituents.

\begin{tabular}{|c|c|c|c|c|c|c|}
\hline Constituents & Characteristics & $\boldsymbol{C}_{\mathbf{0}}$ & $\boldsymbol{C}_{\mathbf{- 1}}$ & $\boldsymbol{C}_{\mathbf{1}}$ & $\boldsymbol{C}_{\mathbf{2}}$ & $\boldsymbol{C}_{\mathbf{3}}$ \\
\hline \multirow{4}{*}{$\begin{array}{c}\text { Ceramic } \\
\text { Silicone nitrite } \\
\mathbf{S i}_{\mathbf{3}} \mathbf{N}_{\mathbf{4}}\end{array}$} & $E(\mathrm{~Pa})$ & $348,4310^{9}$ & 0 & $-3,07010^{-4}$ & $2,16010^{-7}$ & $-8,94610^{-7}$ \\
\cline { 2 - 7 } & $\alpha\left({ }^{\circ} \mathrm{K}^{-1}\right)$ & $5,872310^{-6}$ & 0 & $9,09510^{-4}$ & 0 & 0 \\
\cline { 2 - 7 } & $\rho\left(\mathrm{Kg} \cdot \mathrm{m}^{-3}\right)$ & 2370 & 0 & 0 & 0 & 0 \\
\hline \multirow{3}{*}{$\begin{array}{l}\text { Metal } \\
\text { Stainless steel } \\
\text { SUS304 }\end{array}$} & $\boldsymbol{v}$ & 0,24 & 0 & 0 & 0 & 0 \\
\cline { 2 - 7 } & $\alpha(\mathrm{Pa})$ & $201,0410^{9}$ & 0 & $3,07910^{-4}$ & $-6,53410^{-7}$ & 0 \\
\cline { 2 - 7 } & $\rho\left(\mathrm{Kg}^{-1}\right)$ & $\left.12,33010^{-6}\right)$ & 0 & $8,08610^{-4}$ & 0 & 0 \\
\cline { 2 - 7 } & $v$ & 0,3262 & 0 & $-2,00210^{-4}$ & $3,79710^{-7}$ & 0 \\
\hline
\end{tabular}

Comparison of the Young's modulus variations across the FG beam-cross section for the case of a beam without porosities and the case of a beam with both types of porosity-distributions is plotted in Figure 3. As mentioned above, Young's modulus decreases in both cases of porosity-distributions, only, this decrease is constant in the even-distribution case, and it is more concentrated in the center of beam-section in the uneven-distribution case.

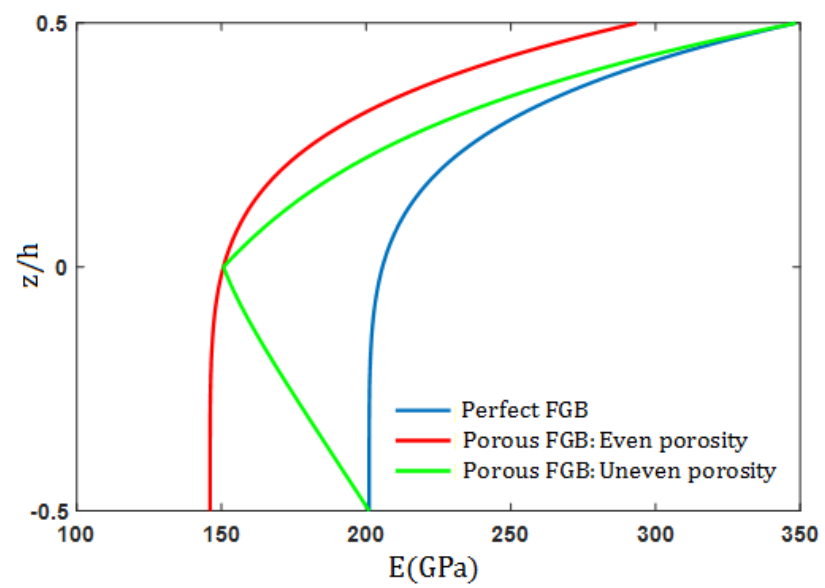

Figure3: Impact of porosity-distribution model on PFG beam-rigidityfor $\zeta=0.2$ and $p=5$.

\subsection{Temperature Distribution}

Thermal environments chosen for this study are modeled by the three fields (uniform, linear and nonlinear sinusoidal) plotted in figure 4. Each environment is described below.

Uniform Distribution(UD): It is assumed in this first case, that the beam is free of constraints, and that the temperature change remains constant according to PFG beam-thickness direction and has as value:

$T=\Delta T+T_{R} ; \quad T_{R}=300^{\circ} \mathrm{K}$ 
$T_{\mathrm{R}}$ is taken as reference.

Linear Distribution(LD): In this second case, temperature-change between both lower and upper beam-faces is linear. This variation across the PFG beam-thickness is defined by [37]:

$T=T_{m}+\Delta T(z / h+0.5) ; \quad \Delta T=T_{c}-T_{m}$

SinusoidalNon-LinearDistribution(SNLD): A sinusoidal nonlinear temperature-distribution is assumed in the third case. It is defined by the following relation [38]:

$$
T(z)=T_{m}+\Delta T[1-\cos ((z / 2 h+1) \pi)] ; \quad \Delta T=T_{c}-T_{m}
$$

$T_{c}$ : Temperature of upper face (Entirely in ceramic). $T_{m}$ : Temperature of lower face (Entirely in metal).

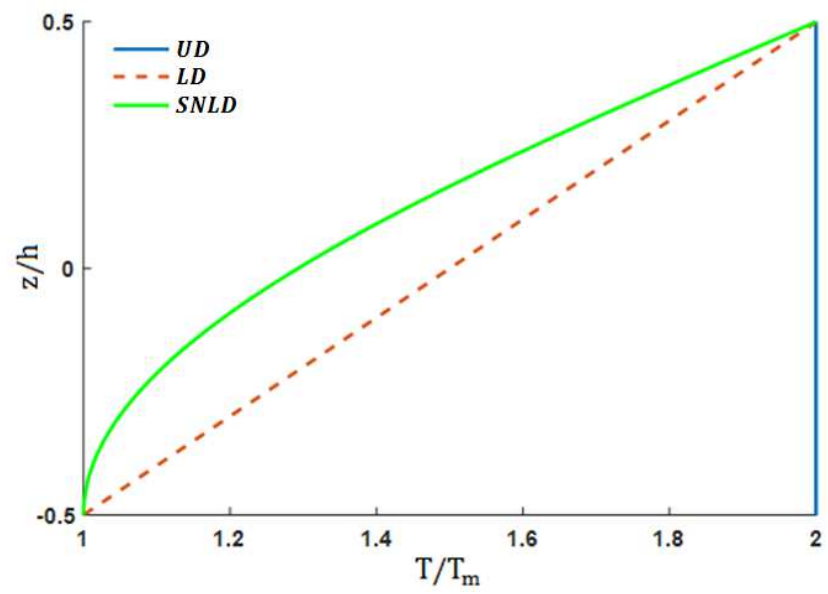

Figure4: Plot of the Three Temperature-Distributions across the PFG Beam-Thickness.

\subsection{Basic Theory}

Displacement field of refined-beam theory is given in a general form as:

$$
\begin{gathered}
U(x, z, t)=u_{0}(x, t)-z \frac{\partial w_{b}}{\partial x}-f(z) \frac{\partial w_{s}}{\partial x} \\
W(x, z, t)=w_{b}(x, t)+w_{s}(x, t)
\end{gathered}
$$

$u_{0}$ : Axial displacement. $w_{b}, w_{s}$ : Bending and Shear displacements respectively.Shear strain-distribution of across the PFG beam-thickness is defined by $f(z)$.

The nonzero strains are given by:

$$
\begin{aligned}
& \varepsilon_{x x}=\frac{\partial U}{\partial x}=\frac{\partial u_{0}}{\partial x}-z \frac{\partial^{2} w_{b}}{\partial x^{2}}-f(z) \frac{\partial^{2} w_{s}}{\partial x^{2}} \\
& \gamma_{x z}=g(z) \frac{\partial w_{s}}{\partial x}, \quad g(z)=\left(1-\frac{d f}{d z}\right)
\end{aligned}
$$

$\varepsilon_{x x}:$ Normal strain. $\gamma_{x z}:$ Transverse shear strain.

According to Hooke's law, expressions of beam-stresses are as follows: 
$\left\{\begin{array}{l}\sigma_{x x}=E \cdot \varepsilon_{x x} \\ \sigma_{x z}=G \cdot \gamma_{x z}\end{array} ; \quad G=\frac{E}{2(1+v)}\right.$

$\sigma_{x x}:$ Axial normal stress. $\sigma_{x z}:$ Shear stress.

First, we calculate the kinetic energy-variation as follows:

$$
\begin{gathered}
\delta \mathcal{K}=\int_{0}^{L} \int_{-h / 2}^{+h / 2} \rho(\dot{U} \delta \dot{U}+\dot{W} \delta \dot{W}) b d z d x \\
=\int_{0}^{L}\left\{I_{0}\left[\dot{u}_{0} \delta \dot{u}_{0}+\left(\dot{w}_{b}+\dot{w}_{s}\right)\left(\delta \dot{w}_{b}+\delta \dot{w}_{s}\right)\right]-I_{1}\left[\dot{u}_{0} \frac{\partial \delta \dot{w}_{b}}{\partial x}+\frac{\partial \dot{w}_{b}}{\partial x} \delta \dot{u}_{0}\right]+I_{2} \frac{\partial \dot{w}_{b}}{\partial x} \frac{\partial \delta \dot{w}_{b}}{\partial x}-J_{1}\left[\dot{u}_{0} \frac{\partial \delta \dot{w}_{s}}{\partial x}+\frac{\partial \dot{w}_{s}}{\partial x} \delta \dot{u}_{0}\right]\right. \\
+J_{2}\left[\frac{\partial \dot{w}_{b}}{\partial x} \frac{\partial \delta \dot{w}_{s}}{\partial x}+\frac{\partial \dot{w}_{s}}{\partial x} \frac{\partial \delta \dot{w}_{b}}{\partial x}\right] \\
\left.+K_{2} \frac{\partial \dot{w}_{s}}{\partial x} \frac{\partial \delta \dot{w}_{s}}{\partial x}\right\} d x
\end{gathered}
$$

where $\left(I_{0}, I_{1}, I_{2}, J_{1}, J_{2}, K_{2}\right)$ are mass inertias, expressed as follows:

$$
\left(I_{0}, I_{1}, I_{2}\right)=\int_{-h / 2}^{+h / 2} \rho\left(1, z, z^{2}\right) b d z, \quad\left(J_{1}, J_{2}, K_{2}\right)=\int_{-h / 2}^{+h / 2} \rho\left(f, z f, f^{2}\right) b d z
$$

Then, we carry out the calculation of the deformation energy-variation as:

$$
\begin{gathered}
\delta \mathcal{U}=\int_{0}^{L} \int_{-h / 2}^{+h / 2}\left(\sigma_{x x} \delta \varepsilon_{x x}+\sigma_{x z} \delta \gamma_{x z}\right) b d z d x \\
=\int_{0}^{L}\left(N \frac{\partial \delta u_{0}}{\partial x}-M_{b} \frac{\partial^{2} \delta w_{b}}{\partial x^{2}}-M_{s} \frac{\partial^{2} \delta w_{s}}{\partial x^{2}}+Q \frac{\partial \delta w_{s}}{\partial x}\right) b d x \\
\left(N, M_{b}, M_{s}\right)=\int_{-h / 2}^{+h / 2}(1, z, f) \sigma_{x x} d z \quad ; \quad Q=\int_{-h / 2}^{+h / 2} g \sigma_{x z} d z
\end{gathered}
$$

The nonzero components of the constraint-resultants $N, M_{b}, M_{s}$ and $Q$ can bespecified as:

$$
\left\{\begin{array}{c}
N \\
M_{b} \\
M_{s} \\
Q
\end{array}\right\}=\left[\begin{array}{cccc}
A & B & B_{s} & 0 \\
B & D & D_{s} & 0 \\
B_{s} & D_{s} & H_{s} & 0 \\
0 & 0 & 0 & A_{s}
\end{array}\right]\left\{\frac{\partial u_{0}}{\partial x},-\frac{\partial^{2} w_{b}}{\partial x^{2}},-\frac{\partial^{2} w_{s}}{\partial x^{2}}, \frac{\partial w_{s}}{\partial x}\right\}^{T}
$$

where $\left(A, B, D, B_{s}, D_{s}, H_{s}, A_{s}\right)$ are the PFG beam-stiffness, defined by :

$$
\begin{aligned}
& (A, B, D)=\int_{-h / 2}^{+h / 2} E\left(1, z, z^{2}\right) d z \quad, \quad\left(B_{s}, D_{s}, H_{s}\right)=\int_{-h / 2}^{+h / 2} E\left(f, z f, f^{2}\right) d z \\
& A_{s}=\int_{-h / 2}^{h / 2} G g^{2} d z
\end{aligned}
$$

Finally potential energy-variation due to applied thermal load denoted by $\overline{N^{T}}$ is computed as follows:

$$
\delta \mathcal{V}=\int_{0}^{L} \overline{N^{T}} \frac{\partial\left(w_{b}+w_{s}\right)}{\partial x} \frac{\partial \delta\left(w_{b}+w_{s}\right)}{\partial x} b d x, \quad \overline{N^{T}}=\int_{-h / 2}^{+h / 2} E \cdot \alpha \cdot \Delta T d z
$$


$\alpha$ :Thermal expansion- coefficient. $\Delta T$ : Difference-temperature determined for each temperature-field.

To ensure the beam-buckling when it is heated, it is necessary to stop the axial displacement of the beam-ends (Hard and Simply Supported HSS-PFGB). This leads to the following boundary conditions:

$$
\text { at } x=0, L: u_{0}=w_{b}=w_{s}=0
$$

To obtain the beam-motion equation, Hamilton principle is applied as follows.

$$
\int_{t_{1}}^{t_{2}}(\delta \mathcal{K}-\delta \mathcal{U}+\delta \mathcal{V}) d t=0
$$

Expressions of $\delta \mathcal{K}, \delta \mathcal{U}$ and $\delta \mathcal{V}$ are replaced into the previous Eq. (17). Then, the integrations by parts are carried out taking into account the boundary conditions. Finally, the following differential equation system is obtained in displacement's terms $u_{0}, w_{b}$ and $w_{s}$.

$$
\begin{gathered}
\delta u_{0}: A \frac{\partial^{2} u_{0}}{\partial x^{2}}-B \frac{\partial^{3} w_{b}}{\partial x^{3}}-B_{s} \frac{\partial^{3} w_{s}}{\partial x^{3}}=I_{0} \ddot{u}_{0}-I_{1} \frac{\partial \ddot{w}_{b}}{\partial x}-J_{1} \frac{\partial \ddot{w}_{s}}{\partial x} \\
\delta w_{b}: B \frac{\partial^{3} u_{0}}{\partial x^{3}}-D \frac{\partial^{4} w_{b}}{\partial x^{4}}-D_{s} \frac{\partial^{4} w_{s}}{\partial x^{4}}-\overline{N^{T}} \frac{\partial^{2}\left(w_{b}+w_{s}\right)}{\partial x^{2}} \\
=I_{0}\left(\ddot{w}_{b}+\ddot{w}_{s}\right)+I_{1} \frac{\partial \ddot{u}_{0}}{\partial x}-I_{2} \frac{\partial^{2} \ddot{w}_{b}}{\partial x^{2}}-J_{2} \frac{\partial^{2} \ddot{w}_{s}}{\partial x^{2}} \\
\delta w_{s}: B_{s} \frac{\partial^{3} u_{0}}{\partial x^{3}}-D_{s} \frac{\partial^{4} w_{b}}{\partial x^{4}}-H_{s} \frac{\partial^{4} w_{s}}{\partial x^{4}}-A_{s} \frac{\partial^{2} w_{s}}{\partial x^{2}}-\overline{N^{T}} \frac{\partial^{2}\left(w_{b}+w_{s}\right)}{\partial x^{2}} \\
=I_{0}\left(\ddot{w}_{b}+\ddot{w}_{s}\right)+J_{1} \frac{\partial \ddot{u}_{0}}{\partial x}-J_{2} \frac{\partial^{2} \ddot{w}_{b}}{\partial x^{2}}-K_{2} \frac{\partial^{2} \ddot{w}_{s}}{\partial x^{2}}
\end{gathered}
$$

Here, Navier' analytical solution is adopted to solve the previous differential equations-system. Displacements are expressed as associations of coefficients representing the unknowns to be determined for each value of " $n$ ", and are given as:

$$
\left[\begin{array}{l}
u_{0}(x, t) \\
w_{b}(x, t) \\
w_{s}(x, t)
\end{array}\right]=\sum_{n=1}^{\infty}\left[\begin{array}{c}
U_{n} \cos (\lambda x) e^{i \omega_{n} t} \\
W_{b n} \sin (\lambda x) e^{i \omega_{n} t} \\
W_{s n} \sin (\lambda x) e^{i \omega_{n} t}
\end{array}\right] ; \quad \lambda=\frac{n \pi}{L}
$$

$\omega_{n}$ : Natural frequency eigenvalue associated with the $n^{\text {th }}$ eigen mode. $U_{n}, W_{b n}, W_{s n}$ : Unknown coefficients to be determined for each value of " $n$ ".

The following equations-system is obtained by replacing the displacements in equations (18) by their expressions in equation (19):

$$
\begin{aligned}
& -A \lambda^{2} U_{n}+B \lambda^{3} W_{b n}-B_{s} \lambda^{3} W_{s n}-\omega_{n}^{2}\left[-I_{0} U_{n}+I_{1} \lambda W_{b n}+J_{1} \lambda W_{s n}\right]=0 \\
& B \lambda^{3} U_{n}+\left(\overline{N^{T}} \lambda^{2}-D \lambda^{2}\right) W_{b n}+\left(\overline{N^{T}} \lambda^{2}-D_{s} \lambda^{4}\right) W_{s n}-\omega_{n}^{2}\left[I_{1} \lambda U_{n}-\left(I_{0}+I_{2} \lambda^{2}\right) W_{b n}-\left(I_{0}+J_{2} \lambda^{2}\right) W_{s n}\right] \\
& =0 \\
& B_{s} \lambda^{3} U_{n}+\left(\overline{N^{T}} \lambda^{2}-D_{s} \lambda^{4}\right) W_{b n}+\left(\overline{N^{T}} \lambda^{2}-H_{s} \lambda^{4}-A_{s} \lambda^{2}\right) W_{s n}-\omega_{n}^{2}\left[J_{1} \lambda U_{n}-\left(I_{0}+J_{2} \lambda^{2}\right) W_{b n}-\left(I_{0}+K_{2} \lambda^{2}\right) W_{s n}\right] \\
& =0
\end{aligned}
$$


The above equations-system can be expressd in the following matrix form:

$\left([\mathrm{K}]-\omega_{n}^{2}[\mathrm{M}]\right)\{\Delta\}=\{0\}$

where,

$$
[\mathrm{K}]=\left[\begin{array}{lll}
a_{11} & a_{12} & a_{13} \\
a_{12} & a_{22} & a_{23} \\
a_{13} & a_{23} & a_{33}
\end{array}\right] ; \quad[\mathrm{M}]=\left[\begin{array}{lll}
m_{11} & m_{12} & m_{13} \\
m_{12} & m_{22} & m_{23} \\
m_{13} & m_{23} & m_{33}
\end{array}\right] ; \quad\{\Delta\}=\left\{\begin{array}{c}
U_{n} \\
W_{b n} \\
W_{s n}
\end{array}\right\}
$$

in which,

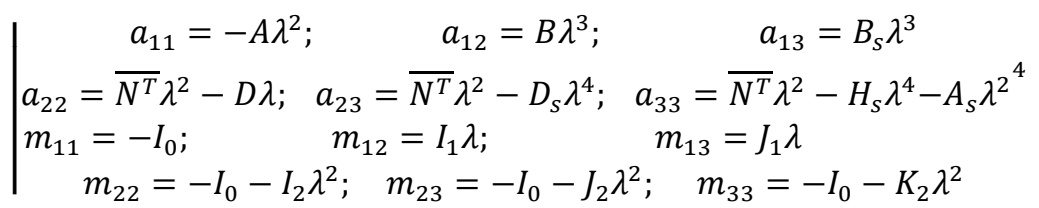

\section{NUMERICAL RESULTS AND DISCUSSIONS}

It is assumed that $T_{m}-T_{R}=5^{\circ} \mathrm{K}$ [39].Expression of the $n^{\text {th }}$ non-dimensional natural frequency isas follows:

$$
\overline{\omega_{n}}=\omega_{n} \frac{L^{2}}{h} \sqrt{\frac{\rho_{m}}{E_{m}}}
$$

To validate method investigated in this work, results obtained here concerning dimensionless fundamental frequencies are summarized in Table 2. These results are compared with those found by the authors [32] and [40], who used different approaches to determine the equations of motion. Farzad Ebrahimi and Ali Jafari [32] applied DTM (Differential Transformation Method), while, Mesut Simsek [40] used Lagrange's equations.It can be confirmed that the obtained results are in good agreement with the results of Refs [32, 40].The shear function used here, to valid numerical results is as follows:

$$
f(z)=\frac{4 z^{3}}{3 h^{2}}(25)
$$

Table 2: Non-Dimensional Fundamental Frequencies of HSS-FGB without Porosities

\begin{tabular}{|c|c|c|c|c|c|c|}
\hline \multirow{2}{*}{$\boldsymbol{L} / \boldsymbol{h}$} & \multirow{2}{*}{ Adopted Method } & \multicolumn{4}{|c|}{$\boldsymbol{p}$} \\
\cline { 2 - 7 } & & $\mathbf{0}$ & $\mathbf{0 . 2}$ & $\mathbf{0 . 5}$ & $\mathbf{1}$ & $\mathbf{2}$ \\
\hline \multirow{3}{*}{5} & DTM [32] & 5.15239 & 4.80599 & 4.41058 & 3.99649 & 3.64468 \\
\cline { 2 - 7 } & Lagrange[40] & 5.15238 & 4.80649 & 4.40828 & 3.99027 & 3.63429 \\
\cline { 2 - 7 } & Navier-solution [Present study] & 5.15267 & 4.80799 & 4.41059 & 3.99039 & 3.62639 \\
\hline \multirow{3}{*}{20} & DTM [32] & 5.46029 & 5.08129 & 4.65109 & 4.20549 & 3.83748 \\
\cline { 2 - 7 } & Lagrange [40] & 5.46028 & 5.08258 & 4.65128 & 4.20499 & 3.83669 \\
\cline { 2 - 7 } & Navier-solution [Present study] & 5.46027 & 5.08149 & 4.65108 & 4.20498 & 3.83608 \\
\hline
\end{tabular}

To highlight the impact of the material-parameter on the frequency-responses, the variation of the dimensionless fundamental frequencies of an HSS-PFGB versus $p$ is plotted in fig.5 for $\zeta=0.2 ; L / h=25$. And to illustrate influence of hot-environment, the beam was exposed to three thermal fields (UD, LD and SNLD). This plot shows that the frequencies decrease when $p$ increases. In fact, Constitution of the beam-material goes from ceramic alone to a ceramicmetal mixture, when $p$ varies from 0 to 10 . By adding metal, beam becomes less rigid because the metal-stiffness is very 
low compared to that of the ceramic. In addition, metal is denser than ceramic, so the beam-mass increases, which makes it more flexible, and justifies the frequencies increase. Effect of the temperature is very clear in this plot since the highest frequencies are obtained when the beam is exposed to a nonlinear sinusoidal thermal field.

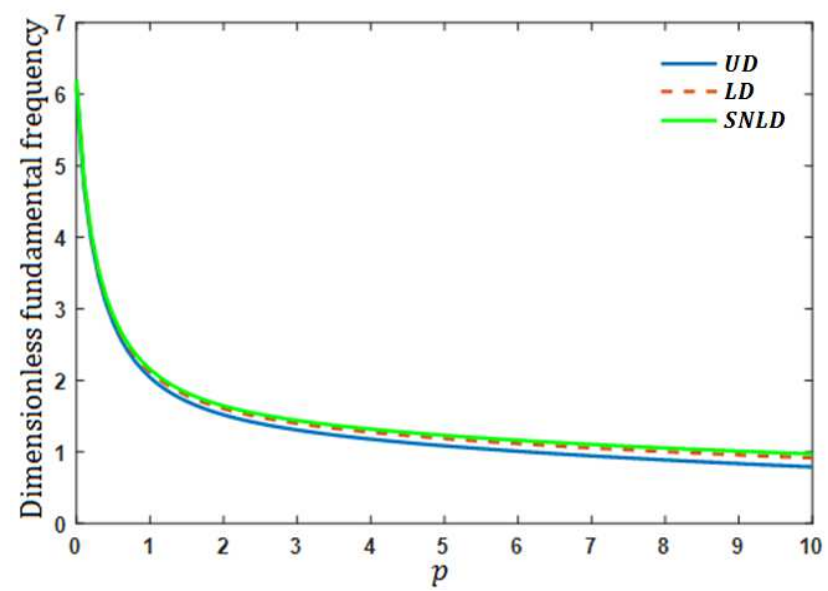

Figure5: Effect of Material-index $(p)$ and Temperature-Distribution on Frequency-responses of HSS-PFGB with Even Porosity-distribution, for $\Delta T=100^{\circ} \mathrm{K}$.

Table3 summarizes fundamental frequencies values obtained for a HSS-PFGB with both porosity-distributions, exposed to three types of hot environments. Some values of $p$ are assumed. These results also reveal, as has been shown previously that, the material-parameter increase drops the frequencies. This impact becomes greater in a nonlinear sinusoidal thermal field with an even porosity-distribution.

Table 3: Non-Dimensional Fundamental Frequencies of HSS-FGB with both porosity-distributions and Exposed to Different Thermal Fields, for Some Values of $p .(L / h=25)$.

\begin{tabular}{|c|c|c|c|c|c|c|}
\hline \multirow{2}{*}{$\Delta T=100^{\circ} K$} & \multirow{2}{*}{$\zeta=0.2$} & \multicolumn{5}{|c|}{$p$} \\
\hline & & $\mathbf{0}$ & 0.5 & 1 & 5 & 10 \\
\hline \multirow{3}{*}{ Even porosity distribution } & UD & 6.0864 & 2.8004 & 2.0415 & 1.0836 & 0.7889 \\
\hline & LD & 6.1582 & 2.8723 & 2.1196 & 1.1858 & 0.9162 \\
\hline & SNLD & 6.1944 & 2.9057 & 2.1551 & 1.2307 & 0.9701 \\
\hline \multirow{3}{*}{ Uneven porosity distribution } & UD & 5.5964 & 2.9409 & 2.2525 & 1.35623 & 1.1006 \\
\hline & LD & 5.6576 & 3.0070 & 2.3239 & 1.4439 & 1.2017 \\
\hline & SNLD & 5.6886 & 3.0380 & 2.3567 & 1.4833 & 1.24632 \\
\hline
\end{tabular}

Figure 6(a) illustrates fundamental frequencies variation versus beam-slenderness for $p=0.5$. Beam with even porosity-distribution is subjected to SNLD. The plot shows a decrease of fundamental frequencies when slenderness ratio increases. Impact of temperature-change on fundamental frequencies is presented in Fig 6(b) for $L / h=20$ and different values of the material index. HSS-PFGB with even porosity-distribution is subjected to UD. As can be observed that, for whatever values of $p$ fundamental frequencies fall when temperature increases until becomes zero. This value corresponds to critical-temperature at which the beam begins to buckle. What explains this phenomenon is the increase in the ductility of the beam material when it's heated. This causes a fall of geometric rigidity of the beam.After this critical value, fundamental frequencies take off; this causes the beam to buckle. As well, the rise in temperature can soften the beam before buckling, and it can become more and softer, when the temperature increases. It is observed also that the temperature which causes the buckling of the beam decreases, when the material-parameter increases. 

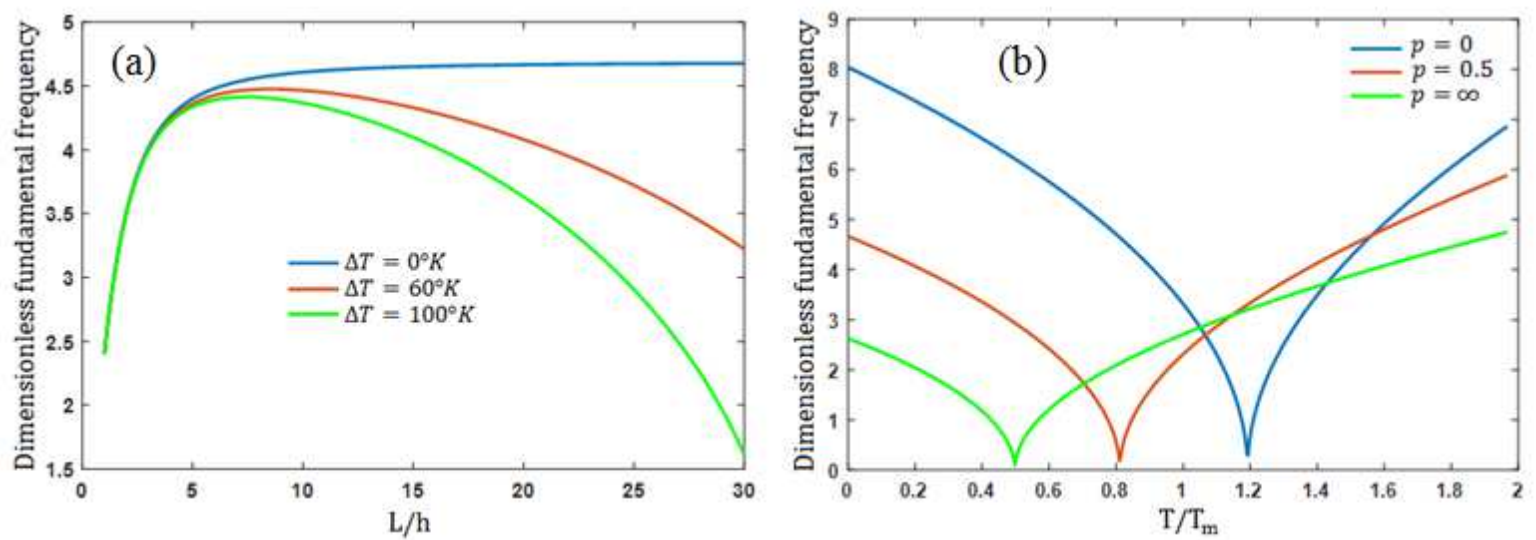

Figure 6: Effect of (a): Slenderness Ratio; (b):Temperature Rise, on Natural Frequencies of HSS-PFGB for $\zeta=0.2$

The variation of fundamental natural frequencies versus temperature for a given slenderness $L / h=20$ and some porosity-parameter is presented in Fig.7 (a), to illustrate the porosity impact on the critical temperature which causes the buckling of PFG beam with even porosity-distribution. This is exposed at a thermal environment whose field is nonlinear sinusoidal. The critical temperature which causes the beam-buckling increases with the increase of the porosity-index since the beam-stiffness increases.

The plot in Figure 7 (b) shows the variation of the fundamental natural frequencies versus the temperature for slenderness ratio $L / h=20$, a porosity-index $\zeta=0.2$. The beam is exposed to three different thermal environments (UD, LD and SNLD) to prove the influence of thermal field on the natural frequencies of the beam. This figure reveals that the beam buckling-temperature in the case of a uniform field loading is important by comparing it with the other thermal loads.

Finally, to show the effect of the beam-slenderness on natural frequencies, Figure 7 (c) presents the variation of the fundamental natural frequencies versus the temperature for $\zeta=0.2$ and some beam-slenderness. The PFG beam with an even porosity-distribution is placed in the thermal environment (SNLD). This figure shows that the beam bucklingtemperature increases when the beam-slenderness decreases. This can be proven experimentally. 

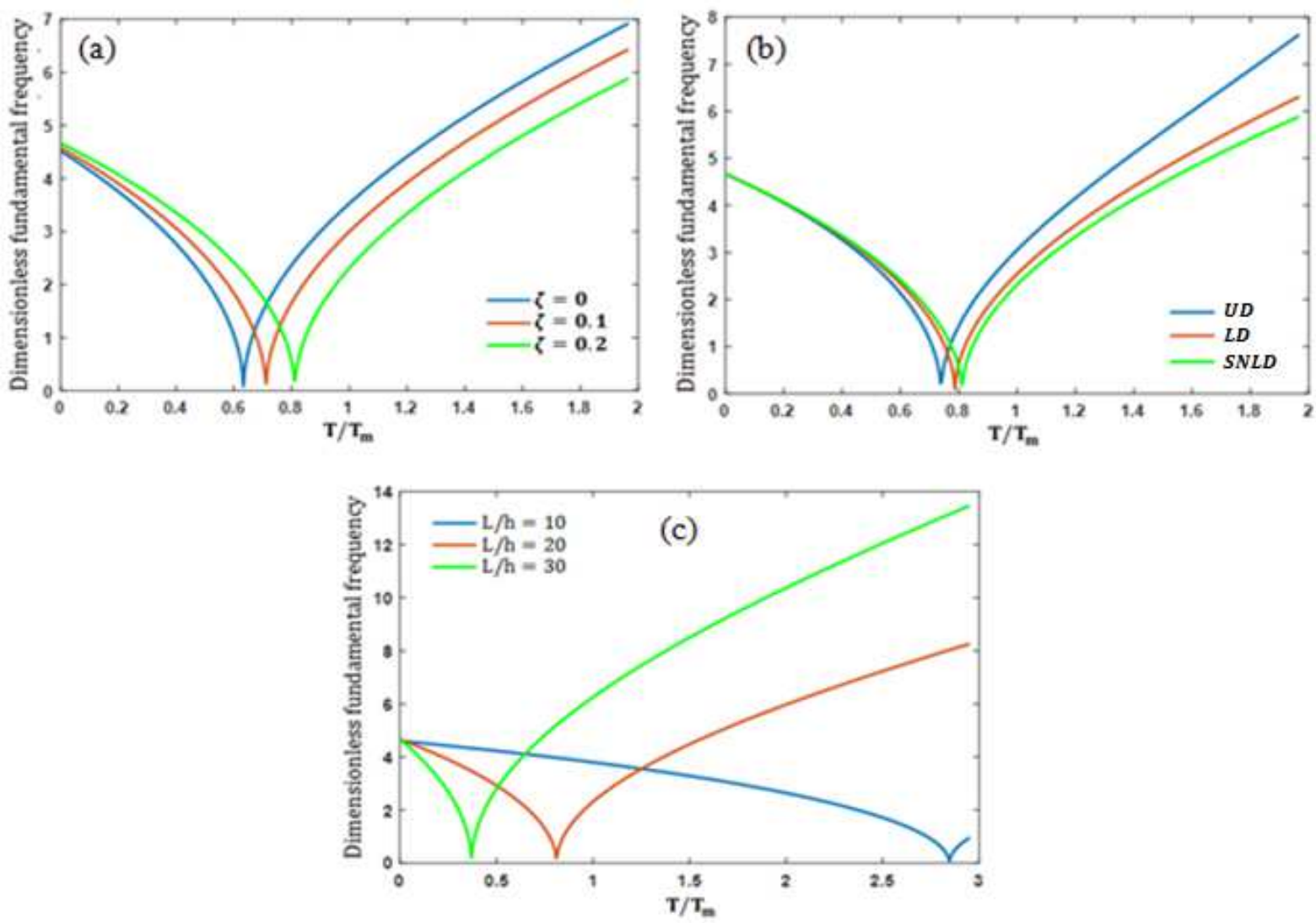

Figure 7: Impact of (a): Porosity-index; (b):Thermal Environment; (c): Slenderness Ratio onThermal Buckling of HSS-PFGB for $\boldsymbol{p}=\mathbf{0 . 5}$.

\section{CONCLUSIONS}

Studying and analyzing the bending behavior of an FG beam containing porosities is the subject of this research. The beam is placed in different thermal-environments with boundary conditions HSS (Hard and Simply Supported) to allow it to buckle.Impacts of thermal-environment, porosity-distribution and beam-slenderness on frequency responses and on beambuckling temperature are discussed. Graphical and analytical results obtained here demonstrate that:

- More material-parameter becomes important, more natural-frequencies increase.

- Over a beam is short more than its natural-frequency is high.

- As temperature-change decreases, natural-frequency increases, because a decrease in temperature leads to an increase in beam-stiffness which retains its mass.

- Thermal-environment, especially SNLD field, enormously affects bending vibration frequency-responses of a PFG beam.

- Porosity-distribution, mainly even distribution, has anexpressive impact on PFG beam-buckling temperature.

- The type of the temperature field has a considerable effect on thermal buckling and frequencies, and the impact is greater in the case of the sinusoidal field compared to the others assumed in this work, uniform and linear.

- To conclude, some parameters as, porosity-index, material-parameter, thermal field shape and porositydistribution, affect beam-buckling in a thermal-environment, and PFG Beam frequency-response. These 
influential parameters must therefore be considered during vibrations analysis of structures exposed to hot environments.

\section{REFERENCES}

1. Koizumi M. FGM activities in Japan. Composites Part B: Engineering 1997; 28 (1-2): 1-4.

2. Sankar BV. “An elasticity solution for functionally graded beams”. Compos Sci Techno 2001; 61(5):689-96.

3. Chakraborty A, Gopalakrishnan S, Reddy JN. "A new beam finite element for the analysis of functionally graded materials". Int J Mech Sci 2003; 45(3):519-39.

4. Chakraborty A, Gopalakrishnan S. "A spectrally formulated finite element for wave propagation analysis in functionally graded beams". Int J Solids Struct 2003; 40(10):2421-48.

5. Aydogdu M, Taskin V. "Free vibration analysis of functionally graded beams with simply supported edges”. Mater Des 2007; 28(5):1651-6.

6. Zhong Z, Yu T. “Analytical solution of a cantilever functionally graded beam”. Compos Sci Techno 2007; 67(3-4):481-8.

7. Kapuria S, Bhattacharyya M, Kumar AN. "Bending and free vibration response of layered functionally graded beams: a theoretical model and its experimental validation”. Compos Struct 2008; 82(3):390-402.

8. Li XF. "A unified approach for analyzing static and dynamic behaviors of functionally graded Timoshenko and EulerBernoulli beams". J Sound Vib 2008; 318(4-5):1210-29.

9. Benatta MA, Mechab I, Tounsi A, Adda Bedia EA. "Static analysis of functionally graded short beams including warping and shear deformation effects". Comput Mater Sci 2008; 44(2):765-73.

10. Sallai BO, Tounsi A, Mechab I, Bachir BM, Meradjah M, Adda BEA. "A theoretical analysis of flexional bending of Al/Al2O3 S-FGM thick beams". Comput Mater Sci 2009; 44(4):1344-50.

11. Sina SA, Navazi HM, Haddadpour H. "An analytical method for free vibration analysis of functionally graded beams”. Mater Des 2009; 30(3):741-7.

12. Şimşek M. "Static analysis of a functionally graded beam under a uniformly distributed load by Ritz method". Int J Eng Appl Sci 2009; 1(3):1-11.

13. Khalili SMR, Jafari AA, Eftekgari A."A mixed Ritz-DQ method for forced vibration of functionally graded beams carrying moving loads". Composite Structures 2010, 92(10): 2497-2511.

14. Kocaturk T, Şimşek M, Akbas SD. "Large displacement static analysis of a cantilever Timoshenko beam composed of functionally graded material”. Sci Eng Compos Mater 2011; 18(1):21-34.

15. Şimşek M, Cansız S. "Dynamics of elastically connected double-functionally graded beam systems with different boundary conditions under action of a moving harmonic load”. Composite Structures 2012; 94(9): 2861-2878

16. Su H, Banerjee JR. "Dynamic stiffness formulation and free vibration analysis of functionally graded beams". Composite Structures 2013; 106: 854-862

17. Mohammad Rezaiee-Pajan, Seyed Mojtaba Hozhabrossadati. "Analytical and numerical method for free vibration of doubleaxially functionally graded beams”. Composite Structures 2016, 152: 488-498.

18. Mergen H.Ghayesh. "Vibration analysis of shear-deformable AFG imperfect beams". Composite Structures 2018, 200: 910920. 
19. Trinh LC, Vo TP, Thai H-T and Nguyen T-K. "An analytical method for the vibration and buckling of functionally graded beams under mechanical and thermal loads". Composites Part B: Engineering 2016; 100: 152-163.

20. El-Megharbel A. "A Theoretical Analysis of FG Beam under Thermal Loading”. World Journal of Engineering and Technology 2016; 4: 437-449.

21. Şimşek M.”Buckling of Timoshenko beams composed of two-dimensional functionally graded material (2D-FGM) having different boundary conditions". Composite Structures 2016, 149: 304-314.

22. Davoodinik AR and Rahimi GH. "Thermal Behavior Analysis of the Functionally Graded Timoshenko's Beam”. IUST International Journal of Engineering Science 2008; 19: 105-113.

23. Ma LS and Lee DW. "A Further Discussion of Nonlinear Mechanical Behavior for FGM Beams under In-Plane Thermal Loading”. Composite Structures 2011; 93: 831-842.

24. Giunta G, Belouettar S and Carrera E. "A Thermal Stress Analysis of Three-Dimensional Beams by Refined One-Dimensional Models and Strong Form Solutions". Applied Mechanics and Materials 2016; 828: 139-171.

25. Zhu J, Yin Z, Lee S, Lai Z and Jeon J. "Fabrication of ZrO2-NiCr functionally graded material by powder metallurgy". Materials Chemistry and Physics 2001; 68(1-3): 130-135.

26. Rezaei AS and Saidi AR. "Application of Carrera Unified Formulation to study the effect of porosity on natural frequencies of thick porous-cellular plates”. Composites Part B: Engineering 2016; 91: 361-370.

27. Wattanasakulpong $N$ and Ungbhakorn $V$. "Linear and nonlinear vibration analysis of elastically restrained ends FGM beams with porosities". Aerospace Science and Technology 2014, 32(1): 111-120.

28. Ebrahimi F and Mokhtari M. "Transverse vibration analysis of rotating porous beam with functionally graded microstructure using the differential transform method". Journal of the Brazilian Society of Mechanical Sciences and Engineering 2015:1435-1444.

29. Wattanasakulpong $N$ and Chaikittiratana A. "Flexural vibration of imperfect functionally graded beams based on Timoshenko beam theory: Chebyshev collocation method”. Meccanica 2015: 1331-1342.

30. Ait Atmane H, Tounsi A and Bernard F. "Effect of thickness stretching and porosity on mechanical response of a functionally graded beams resting on elastic foundations”. International Journal of Mechanics and Materials in Design 2017; 13(1): 7184.

31. KHAN, S. A., SHAHZER, M., SULTAN, D., \& ALI, R. (2019). Thermal and solutalbuoyancyeffects on mixing of opposedlaminar jets in a two-dimensional passive mixer at a higher Reynolds number. Int. J. Mech. Prod. Eng. Res. Dev., 9(3), 923-930.

32. Ebrahimi $F$ and Salari E. "Thermo-mechanical vibration analysis of nonlocal temperature-dependent FG nanobeams with various boundary conditions". Composites Part B: Engineering 2015; 78: 272-290.

33. Ebrahimi $F$ and Jafari A. "Thermo-mechanical vibration analysis of temperature-dependent porous FG beams based on Timoshenko beam theory”. Structural Engineering and Mechanics 2016,59(2): 343-371.

34. Ebrahimi $F$ and Jafari A. "A Higher-Order Thermomechanical Vibration Analysis of Temperature-Dependent FGM Beams with Porosities". Journal of Engineering 2016.

35. Reddy JN and Chin CD. "Thermomechanical analysis of functionally graded cylinders and plates". International Journal of Solids and Structures 1998; 21:593-626. 
36. Touloukian YS. "Thermophysical properties of high temperature solid materials. Volume 4. Oxides and Their Solutions and Mixtures. Part I. Simple Oxygen Compounds and Their Mixtures”. DTIC Document 1966.

37. Chi S and Chung YL. "Cracking in coating-substrate composites of multi-layered and sigmoid FGM coatings". Engineering Fracture Mechanics 2003, 70 (10): 1227-1243.

38. Mahrous, A. (2013). Thermal performance of PCM basedheatsinks. Int J Mech Eng, 2 (4).

39. Kiani $Y$ and Eslami MR. "An exact solution for thermal buckling of annular FGM plates on an elastic medium. Composite Part B: Engineering 2013; 45(1):101-110.

40. Na KS and Kim JH. "Three-dimensional buckling analysis of functionally graded materials". Composite Part B: Engineering 2004; 35(5): 429-437.

41. Yang $J$ and Shen HS. "Nonlinear bending analysis of shear deformable functionally graded plates subjected to thermomechanical loads under various boundary conditions". Composites Part B: Engineering 2003; 34:103-115.

42. Şimşek M. "Fundamental frequency analysis of functionally graded beams by using higher-order beam theories". Nuclear Engineering and Design, 2010; 240(4): 697-705. 